\title{
Large Solid Angle Silicon Drift Detectors for EDX Analysis in TEM
}

\author{
A. Niculae ${ }^{1}$, M. Bornschlegl ${ }^{1}$, R. Eckhardt ${ }^{1}$, J. Herrmann ${ }^{1}$, S. Jeschke ${ }^{1}$, G. Krenz ${ }^{1}$, A. Liebel ${ }^{1}$, G. Lutz ${ }^{2}$, \\ H. Soltau ${ }^{1}$, L. Strüder ${ }^{2}$ \\ ${ }^{1}$ PNDetector GmbH, Emil-Nolde-Str.10, 81735 München, Germany \\ 2 PNSensor GmbH, Römerstr. 28, 80803 München, Germany
}

Transmission Electron Microscopes (TEM) have become over the last years very powerful analytical tools for a various applications ranging from material analysis in the semiconductor industry to biological cell microscopy in life sciences. Very often the TEM instruments are equipped with Energy Dispersive X-Ray spectroscopy (EDX) tools, which in STEM mode enable fast and accurate elemental analysis on atomic level.

As the samples used for analysis in TEM are typically few tens of nanometers thin, the fluorescence yield available for the EDX analysis is quite low and therefore EDX detectors with high geometrical collection efficiency (solid angle) are needed. As a pioneer in the field of the Silicon Drift Detectors and leading the market of EDX detector modules for microanalysis, the company PNDetector in Munich has been continuously working on optimized detector configurations for specific applications. In the recent years various detector geometries and packaging solution specially dedicated to EDX microanalysis in TEM have been successfully developed. The primary goal here is to tailor the detector geometry and optimize the package dimensions in such a way that the detector covers the highest possible solid angle allowing for fast analysis time.

Figure 1 shows the calculated solid angle as a function of the detector area $A$ (round-shaped detector) and the distance from the sample $d$. As it can be clearly seen, in order to achieve a large solid angle it is much more effective trying to decrease the distance between detector and sample than increasing the detector area. As the space around a TEM pole piece is very limited, bringing the detector very close to the sample becomes a big challenge and thus, an intelligent approach in designing the detector is mandatory.

In this paper we present an SDD configuration which successfully overcomes the difficulty mentioned above - this is the so-called SDD "Racetrack" configuration. The SDD chip has a total area of $100 \mathrm{~mm}^{2}$ with the special shape of a racetrack - see Figure 2. This special geometry enables the positioning of the detector at short distances to the sample, leading to solid angles coverage close to $1 \mathrm{sr}$ (depending on the pole-piece gap and geometry).

The spectroscopic performance of the Racetrack SDD is shown in Figure $2 b$ where the energy resolution is plotted as a function of shaping time (or maximum input count rate). Due to the very low leakage current levels, the detector shows very good performance already at $-20^{\circ} \mathrm{C}$, the optimum energy resolution of about $129 \mathrm{eV}$ is reached at $1-2 \mu$ s shaping time and $-30^{\circ} \mathrm{C}$. The detector module can be used in a windowless configuration allowing the maximum detection efficiency of light elements down to Boron or can be hermetically closed with a light element window. Measurements with an optimized version of the detector, featuring the optimized FET - the so called SDD ${ }^{\text {plus }}$ FET will be presented as well and the results will be discussed. 

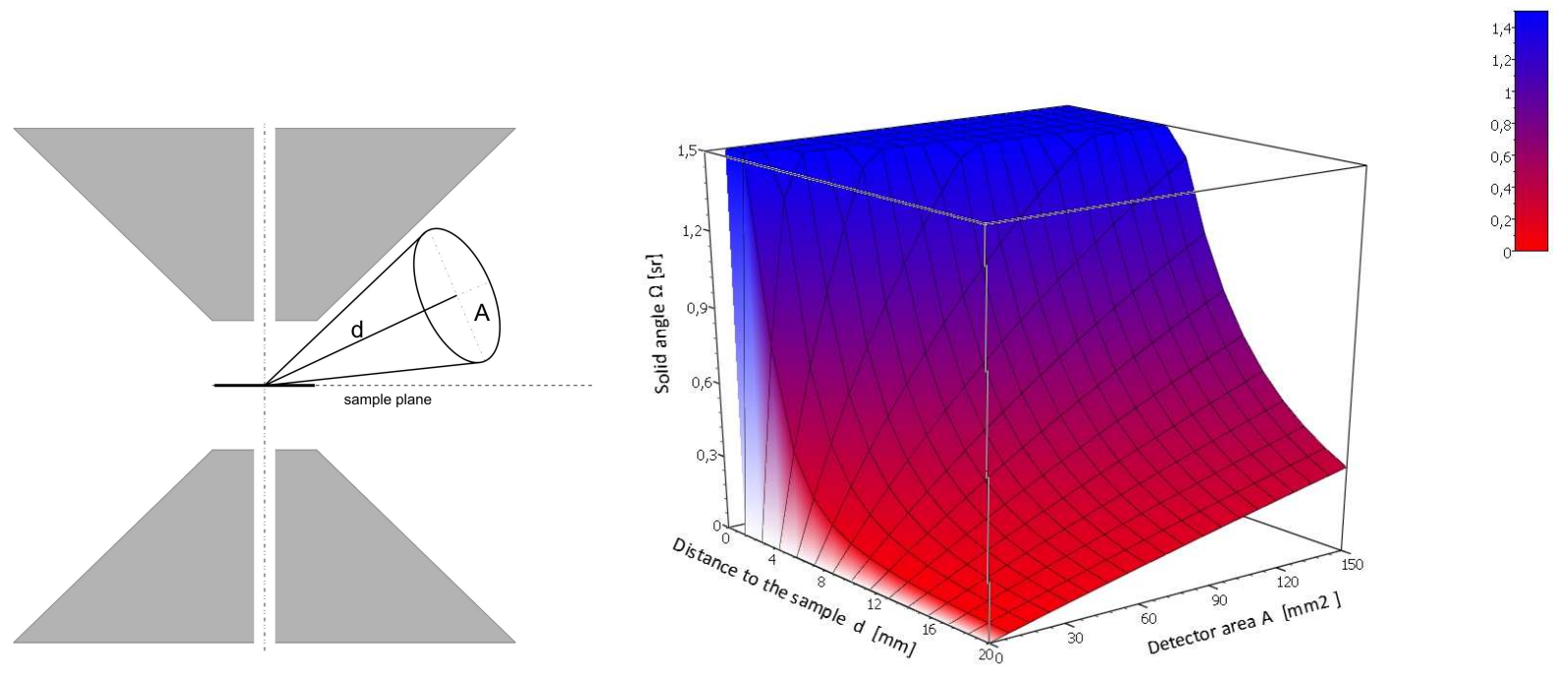

Fig. 1 Calculated solid angle of a round shaped detector in a TEM as a function of the detector area and distance to the sample.

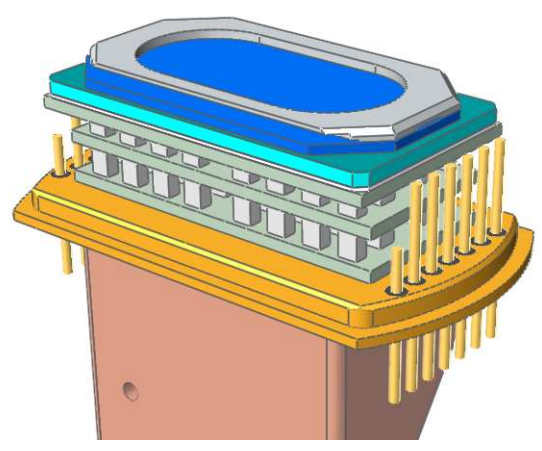

(a)

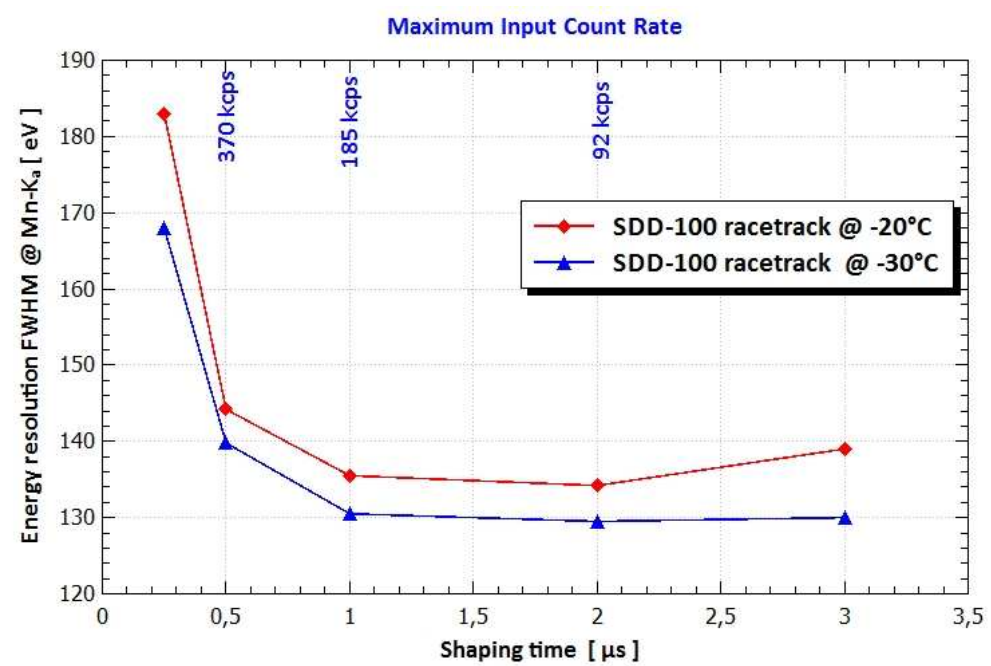

(b)

Fig. 2: (a) SDD "Racetrack" detector module configuration. (b) Spectroscopic performance at various count rate values and moderate cooling temperatures. 